\title{
Conceptual Design and Research on Automatic Feeding Device Based on Linear Servo Motor
}

\author{
Liang Han $^{\mathrm{a}}$, Kaige $\mathrm{Yan}^{\mathrm{b}}$, Junjing Wang ${ }^{\mathrm{c}}$ \\ School of Mechanical Engineering, Southeast University, Jiangning District, Nanjing 211189, China \\ amelhan@seu.edu.cn, bkaige_yan@163.com, cwjjgogo@126.com
}

Keywords: Automatic Feeding Device; Linear Servo Motor; Velocity Planning

\begin{abstract}
The automatic feeding device occupies the irreplaceable position in the development of industrial automation. An automatic feeding device is often required to convey parts to complete a series of processes in a modern production line. But the traditional automatic feeding device has problems of large volume, high noise and low efficiency. It has reduced the efficiency of production line. So it is necessary to make innovation for the automatic feeding system. A new type of automatic feeding device based on linear servo motor is proposed in the paper. This device overcomes some disadvantages of the traditional automatic feeding device.
\end{abstract}

\section{Introduction}

A production line often includes automatic feeding device which makes the disorderly parts deliver to the next procedure neatly and accurately. Automatic feeding device is a kind of conveying mechanisms, which runs automatically according to the specified requirements and procedures, and the operators only need to determine the control requirements and procedures instead of directly operating the conveying mechanism. Automatic feeding device which generally includes inspection mechanisms and conveying mechanisms, etc. is mainly used to convey finished or semi-finished products. It also cooperates with next working procedures to make production automatic.

At present, automatic feeding devices widely applied include mechanical vibratory screen, rotary funnel, elevating feeding device, centrifugal funnel, vibratory feeder, etc. Due to disadvantages of these mechanisms, some problems exist in the traditional automatic feeding device generally. For instance, mechanical vibratory screen has large size, high noise, serious abrasion and low efficiency; Rotary funnel conveys parts on the rotating plate, which often makes parts scratched. These defects affect the quality of parts feeding seriously and cut down the efficiency of production line.

With the development of industrial production, more factories requires better environment, lower cost and higher efficiency of production equipment, so as to make their products more competitive. Linear servo motor is famous for its small size, low cost, good flexibility, low noise and simple structure urgently ${ }^{[1-6]}$. According to the needs of the development of automation equipment, a novel automatic feeding device based on linear servo motor is designed in this paper. The device has the advantages of simple structure, long lifespan, low noise and easy control, which is driven by linear servo motor. The automatic feeding device based on linear motor is described in detail below.

\section{Introduction to the linear motor}

With the rapid development of automatic control and microcomputer technology, higher requirements of positioning precision of all kinds of automatic control system are put forward. In this case, the traditional rotating motor and a set of conversion mechanism of linear motion device drivers can not satisfy the demands of modern control system. Therefore, many countries are researching and developing linear motor, and linear motor is applied more and more widely. Linear motor is a device which converts electrical energy into linear motion directly without any intermediate conversion mechanisms. As shown in Fig.1, the linear motor can be seen as a rotary motor sectioned along the radius, and unfold to plane. 


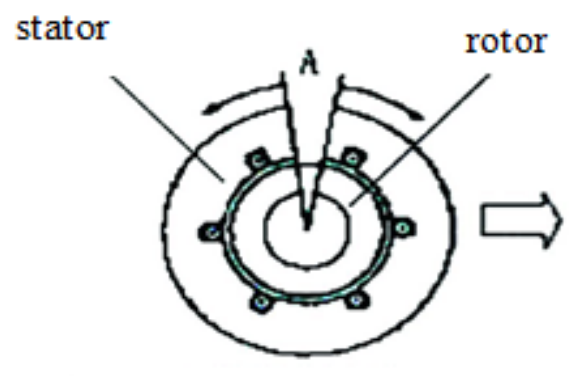

a) Cut along the radial

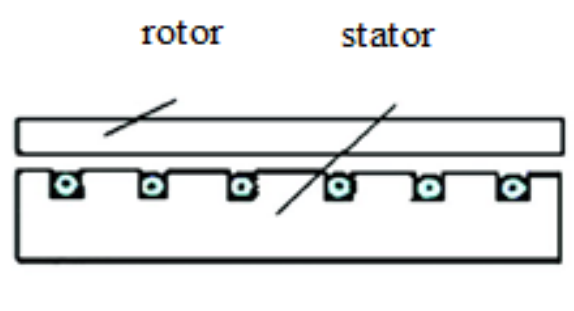

b) Unwind the circle into a straight line

Fig. 1 structure of rotary motor and linear motor

Linear motors can be classified into the following: D.C. motors, induction motors, synchronous motors and stepping motors, etc. [7-8] Compared with rotary motor, the linear motor has many advantages. Firstly, the structure of the linear motor is simple. Because the linear motor does not need the additional device to convert rotation into linear motion, the structure of the system is greatly simplified and its weight and volume are decreased. Secondly, the linear motor has high position accuracy. Linear motor can realize direct driving which can eliminate the positioning error of intermediate mechanisms, so it has the high positioning accuracy. Thirdly, the linear motor has quick response, high sensitivity and good trackability. Linear motor always maintains a certain gap between the stator and rotor, which eliminates friction resistance between stator and rotor, thus the system's sensitivity, rapidity and trackability are improved greatly. Lastly, the linear motor works safely and enduringly. And it has less breakdown and needs less maintenance, thus it can work safely, reliably and enduringly [9-12].

\section{Design of automatic feeding device based on linear servo motor}

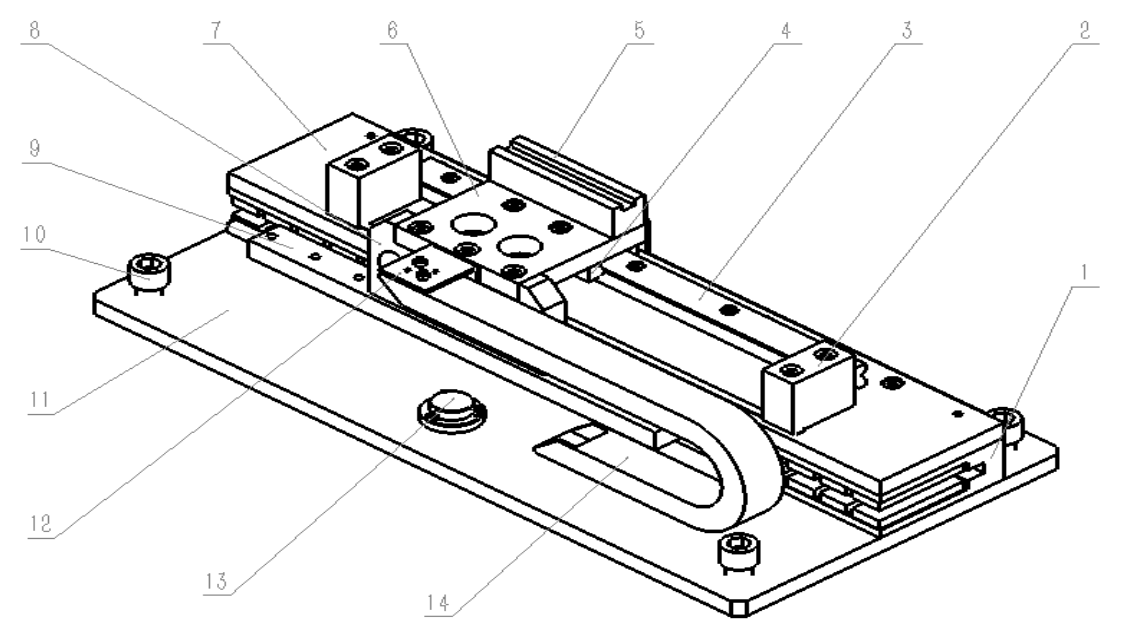

1. Magnet yokes; 2. Limit stop; 3. Linear guide; 4. Slider; 5. Feeder trough; 6. Supporter (H); 7. Mounting frame; 8. Supporter (V); 9. Linear motor rotor; 10. Adjusting bolts; 11. Base;

12. Cable protector connecter; 13 . Level; 14 . Cable protector;

Fig. 2 Structure of the automatic feeding device based on linear servo motor

The structure of automatic feeding device based on linear motor is shown in Fig. 2. The trough is fixed on the linear motor; so the movement of trough can be controlled by the linear motor rotor.

The principle of the automatic feeding device: Based on the characteristics of linear motor, such as quick response, low noise, large force and high accuracy, the trough can move with high acceleration precisely. Through the control of linear motor, the trough fixed on the rotor moves forward a certain distance slowly, and the workpiece on the trough moves the same distance. Then the trough moves back quickly, but the workpiece moves back a short distance because of its sliding on the trough. When the trough moves repeatedly, the workpiece can run forward smoothly, and the higher the frequency of the movement is, more rapidly the workpiece moves. 


\section{Velocity planning and workpiece modeling}

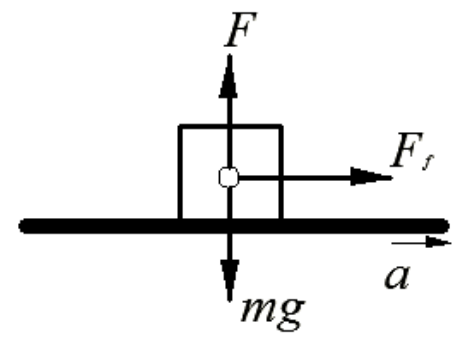

(1)

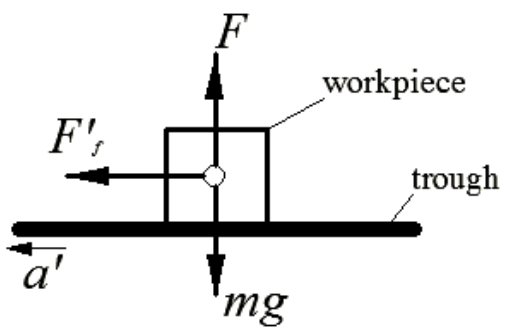

(2)

Fig. 3 workpiece modeling and analysis
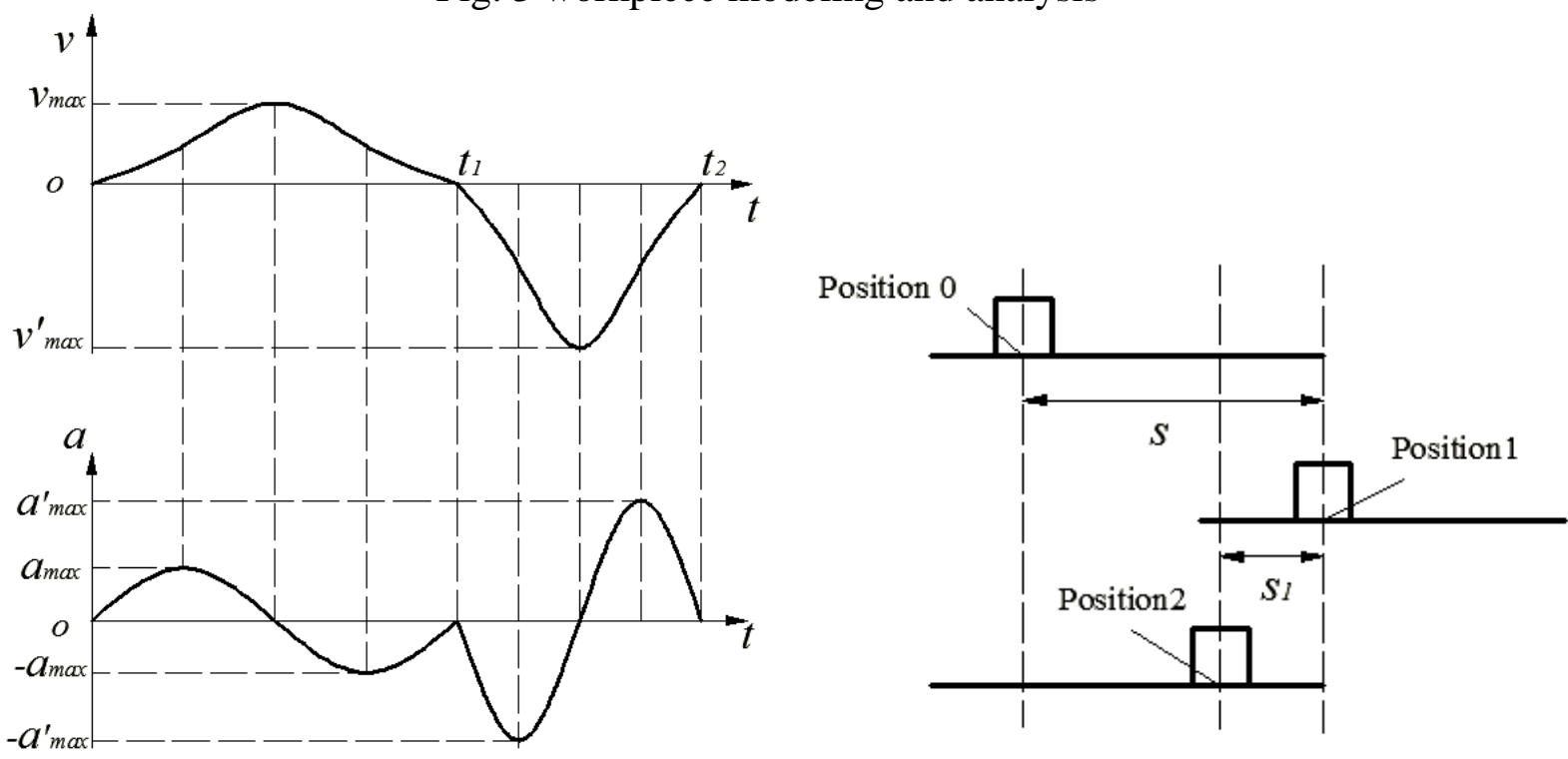

Fig. 4 The movement of workpiece and velocity planning of the linear motor

When the trough moves with positive and negative acceleration, force analysis of the workpiece is shown in Figure 3. It is assumed that the mass of workpiece is $m$ and the coefficient of sliding friction between the workpiece and the trough is $f$. When the trough moves with a positive acceleration and the workpiece keep static relative to the trough, the friction is $F_{f}=m a$ which is less than the sliding friction $m g f$, so the requirement of the trough acceleration is $a<g f$. When the trough moves with a negative acceleration and the workpiece slides on the trough, the friction is $F_{f}{ }^{\prime}=m g f$, so the requirement of the trough acceleration is $a>g f$.

Working process of linear motor automatic feeding device: as shown in Fig. 4(left), after the device is started, by controlling the linear servo motor, the trough moves at a certain distance $S$ with positive sine acceleration in which $a_{\max }<g f$ is required. Because of no sliding between the workspice and the trough, the workspice moves the same distance $S$ and it moves from position 0 to position 1 . When the trough moves back with negative sine acceleration, due to $a_{\text {max }}^{\prime}>>g f$, the workpiece slides on the trough and displacement $s_{1}<s$. So the displacement of the workpiece is $\left(s-s_{1}\right)$ in this period. The velocity and acceleration are shown in Fig. 4(right), and the trough moves with sine acceleration in the forward and backward movements, so the system can run smoothly and has no impact.

\section{Conclusion}

A new type of automatic feeding device based on linear servo motor is proposed in the paper. 
Depending on servo driver and linear grating, it is easy to control the linear motor to achieve high position accuracy. And because of no additional mechanisms to convert rotary motion to linear motion or leaf springs and electromagnets which are significant in traditional feeder, the automatic feeding device based on linear servo motor has advantages such as simple and compact structure, low noise and easy control. The system overcomes some disadvantages of traditional automatic feeding device and has high research value and application prospect.

\section{Acknowledgement}

This work is supported by NSFC, project No.: 51275087.

\section{Reference}

[1] Liu Bin. Automatic production line installation and adjustment [M].Chemical industry press, Beijing, 2012.

[2] Chen Meng, Jin Guolong. Operation and maintenance of automatic production line [M].China water and power press, Beijing, 2012.

[3] Wu Mingliang. Automatic production line technology [M]. Chemical industry press, Beijing, 2011.

[4] Han Liang, Xu Weiliang. Research status and prospect of vibratory feeder [J].Equipment for electronic products manufacturing, 1999.

[5] Gao Hui. A study on key technologies for commercialization of decoupled vibratory feeder [D]. Southeast university, Nanjing, 2008.

[6] Y.M.Li. Research on a novel vibration bowel-feeder and control system [D]. Tianjin University, Tianjin, 2005.

[7] Qinfen Lu, Yanxin Li, Investigation of forces in linear induction motor under different slip frequency for low-speed maglev application [J]. IEEE Transactions on energy conversion, Vol. 28, No.1, 2013.

[8] Brahim Bessaih, Abdelkrim Boucheta, Speed control of linear induction motor considering end-effects compensation using rotor time constant estimation [J].2012.

[9] Ye Yunyue. Linear driving devices and systems [M]. Metallurgical industry press, Beijing, 2000

[10] Ye Yunyue. Linear motor technical manual [M]. China machine press, Beijing, 2003.

[11] P.-K. Huang. Recurrent fuzzy neural network controlled linear induction motor drive based on genetic algorithm [D], Chung Yuan Christian University, Taiwan, 2002.

[12] C.-Y. Hung, Adaptive control for rotary induction motor and linear induction motors [D], Chung Yuan Christian University, Taiwan, 2001. 